\section{Cerebral venous outflow and cerebrospinal fluid dynamics}

\author{
Clive B. Beggs \\ Medical Biophysics Laboratory, \\ University of Bradford, UK
}

\section{Abstract}

In this review, the impact of restricted cerebral venous outflow on the biomechanics of the intracranial fluid system is investigated. The cerebral venous drainage system is often viewed simply as a series of collecting vessels channeling blood back to the heart. However there is growing evidence that it plays an important role in regulating the intracranial fluid system. In particular, there appears to be a link between increased cerebrospinal fluid (CSF) pulsatility in the Aqueduct of Sylvius and constricted venous outflow. Constricted venous outflow also appears to inhibit absorption of CSF into the superior sagittal sinus. The compliance of the cortical bridging veins appears to be critical to the behaviour of the intracranial fluid system, with abnormalities at this location implicated in normal pressure hydrocephalus. The compliance associated with these vessels appears to be functional in nature and dependent on the free egress of blood out of the cranium via the extracranial venous drainage pathways. Because constricted venous outflow appears to be linked with increased aqueductal CSF pulsatility, it suggests that inhibited venous blood outflow may be altering the compliance of the cortical bridging veins.

\section{Introduction}

Traditionally, the cerebral venous drainage system has been viewed simply as a network of collecting vessels channeling blood from the brain to the heart; with the result its regulatory role has tended to be over-looked. However, in recent years there has been renewed interest in the cerebral venous drainage system, because of the discovery of the vascular syndrome chronic cerebrospinal venous insufficiency (CCSVI), ${ }^{1}$ which is characterized by restricted cerebral venous outflow and increased hydraulic resistance to blood flow back to the heart. ${ }^{2}$ Although the subject of CCSVI has been mired with controversy, ${ }^{3}$ with many disputing the validity of the syndrome, ${ }^{4-6}$ there is increasing evidence that venous drainage anomalies may be associated with physiological changes in the intracranial space. ${ }^{7,8}$ This has precipitated renewed interest in the role that venous anomalies might play in neurological disease, ${ }^{9}$ something which has highlighted the close link between the venous drainage system and the dynamics of the cerebrospinal fluid (CSF) system. ${ }^{10}$ In this review we investigate the link between restricted cerebral venous outflow and the biomechanics of the CSF system.

\section{Intracranial fluid volume regulatory mechanism}

Being encased in a rigid enclosure, the brain employs a complex intracranial fluid regulatory mechanism to control the pulsatility of blood flow through the cerebral vascular bed. ${ }^{11-13}$ This system utilizes a sophisticated windkessel mechanism to compensate for the transient increases in arterial blood volume that occur during systole, by displacing an approximately equal volume of CSF out of the cranium into the spinal column ${ }^{14}$ (Figure 1). As such, the system maintains Monro-Kellie homeostasis and ensures that the flow of blood through the cerebral capillary bed is smooth and non-pulsatile in healthy young adults. ${ }^{11,15}$ The whole system is driven by volumetric changes in the arterial pulse, which are transferred to the CSF, causing it to pulse backwards and forwards across the foramen magnum (FM). Although in healthy young adults blood flow through the cerebral capillary bed is normally free of any pulse, by the time it reaches the dural sinuses it once again exhibits pulsatile characteristics. ${ }^{11,16}$ This suggests that the CSF pulse interacts with the venous flow somewhere in the cranium to regulate blood outflow. While this mechanism has generally been thought to be a passive interaction, ${ }^{10}$ recent evidence has emerged to suggest that active venoconstriction of the large extracranial veins may also play a part in the regulatory process. ${ }^{17}$

Deeper insights into the dynamics of the intracranial fluid system can be gained by considering how the fluid flows in and out of the cranium vary over the cardiac cycle. Transient arterial, venous, and CSF flows in and out of the cranium are illustrated in Figure 2, which shows the cervical pulses for a typical healthy individual. ${ }^{16}$ From this it can be seen that the system is driven by the arterial pulse, which as it enters the cranium during systole greatly increases the volume of blood in the pial arteries. ${ }^{18}$ This peaks at about 0.23 of the cardiac cycle and is closely followed by the peak in CSF flow through the FM, which occurs at 0.28 of the cardiac cycle. Finally, in late systole at
Correspondence: Clive B. Beggs, Medical Biophysics Laboratory, School of Engineering, University of Bradford, Bradford, West Yorkshire BD7 1DP, United Kingdom.

Tel.: +44.0.1274.233679 - Fax: +44.0.1274.234124

E-mail: c.b.beggs@bradford.ac.uk

Key words: cerebral venous drainage, cerebrospinal fluid, chronic cerebrospinal venous insufficiency, intracranial pressure, normal pressure hydrocephalus, multiple sclerosis.

Acknowledgments: Clive Beggs received a travel grant from the Annette Funicello Research Fund for Neurological Diseases.

Received for publication: 7 August 2013.

Revision received: 27 0ctober 2014.

Accepted for publication: 4 November 2014.

This work is licensed under a Creative Commons Attribution 3.0 License (by-nc 3.0).

@CCopyright C.B. Beggs, 2014

Licensee PAGEPress, Italy

Veins and Lymphatics 2014; 3:1867

doi:10.4081/vl.2014.1867

about 0.35 of the cardiac cycle, there is a peak in the venous blood flow leaving the cranium. Figure 2 also shows the CSF pulse in the Aqueduct of Sylvius (AoS), which in comparison to the cervical CSF pulse, exhibits a much smaller amplitude and is out of phase.

From Figure 2 it can be seen that during diastole there is a decrease in the venous blood flow rate leaving the cranium. Given that blood flow through the cerebral capillary bed remains relatively constant throughout the cardiac cycle, this implies that during diastole, venous blood is being stored somewhere in the cranium, only to be rapidly ejected during systole. While the physiological mechanisms associated with this strange phenomenon are poorly understood, it is known that approximately $70 \%$ of intracranial blood volume is located within the venous compartments, ${ }^{19}$ many of which are thin-walled veins that can readily expand and collapse with small changes in transmural pressure. ${ }^{20,21}$ It is therefore likely that blood is stored in these vessels during diastole. A number of researchers have reported the presence of regulatory sphincters, ${ }^{22,23}$ which control the discharge from these veins into the superior sagittal sinus (SSS), and it has been postulated that constriction of these sphincters causes the cortical veins to engorge and puff out, before periodically discharging into the $\mathrm{SSS}^{23}$ Evidence supporting this hypothesis comes from Greitz ${ }^{24}$ and Nakagawa et al., ${ }^{25}$ who both observed the pulsatile compression of cortical bridging veins by the sub-arachnoid CSF. 


\section{Cerebrospinal fluid bulk flow}

In addition to the CSF pulse, there is a slow bulk flow of CSF from the choroid plexus (CP) to the SSS, via the arachnoid villi (AV), driven by the pressure gradient between the two. While it used to be assumed that all the CSF was absorbed through the AV into the SSS, $26-28$ it is now thought that some CSF drains to the lymph nodes via nasal lymphatics. ${ }^{29}$ In animals, as much as $50 \%$ of CSF drains to the lymph nodes, ${ }^{30,31}$ whereas in adult humans a greater proportion appears to drain directly into the venous blood via the $\mathrm{AV},,^{29,31}$ with lymphatic drainage playing only a minor role. ${ }^{29}$ The SSS acts as a collecting vessel for CSF from the sub-arachnoid space (SAS). CSF absorption into the SSS via the $\mathrm{AV}$, which has been measured in the range $4.5-9.4 \mathrm{~mm}^{3} / \mathrm{s}$ in healthy individuals, ${ }^{26}$ is very susceptible to changes in the pressure difference between the SAS and SSS. ${ }^{26-28}$ In a study involving 100 healthy adults, Ekstedt ${ }^{26}$ demonstrated that there is a linear relationship between this pressure difference and CSF absorption through the $\mathrm{AV}$, with the average rate of absorption being $2.397 \mathrm{~mm}^{3} / \mathrm{s} / \mathrm{mmHg}$. They measured the mean CSF pressure in the SAS [i.e. the intracranial pressure (ICP)] as being $10.35 \mathrm{mmHg}$ when supine, and calculated that the mean pressure in the SSS was $7.57 \mathrm{mmHg}$, which equates to a mean pressure drop of 2.78 mmHg across the AV.

CSF is produced in the $\mathrm{CP}$, which are located in the walls of the third, fourth and lateral ventricles. The endothelium of the CP is leaky, with no tight junctions, allowing the transfer of fluid (water) between the blood vessels and the $\mathrm{CSF}^{32} \mathrm{~A}$ number of researchers have attempted to quantify CSF production rates in humans. Cutler et al. ${ }^{27}$ in a study involving children with sclerosing panencephalitis and Pontine glioma, measured the mean rate of formation of CSF to be $5.83 \mathrm{~mm}^{3} / \mathrm{s}$. In a similar study, Lorenzo et al..$^{33}$ found the mean CSF production rate in healthy children to be 6.00 $\mathrm{mm}^{3} / \mathrm{s}$. It is possible to obtain a rough estimate of the CSF production rate by monitoring the flow of CSF through the AoS and calculating the difference between the net negative CSF flow (NNF) in the caudal direction and the net positive flow (NPF) towards the third ventricle. Using this methodology, Magnano et al..$^{34}$ found the bulk aqueductal CSF flow in healthy adults to be $7.1 \mathrm{~mm}^{3} /$ beat (approximately 8.28 $\mathrm{mm}^{3} / \mathrm{s}$ ), whereas Beggs et al. ${ }^{7}$ and Gorucu et $a l .{ }^{35}$ in similar studies found mean flow to be $4.0 \mathrm{~mm}^{3} /$ beat (approximately $4.65 \mathrm{~mm}^{3} / \mathrm{s}$ ) and $2.17 \mathrm{~mm}^{3} / \mathrm{s}$, respectively. Given that measured CSF production rates appear to be of similar magnitude to absorption rates through the $\mathrm{AV}$, it suggests that lymphatic drainage of CSF plays only a relatively minor role in humans.

\section{Link between venous outflow and cerebrospinal fluid dynamics}

A number of studies have linked constricted venous outflow with changes in the dynamics of the cerebrospinal fluid system. ${ }^{7,836}$ Under normal circumstances, in healthy individuals the CSF NPF per heartbeat is slightly less than the CSF NNF, with the mathematical difference between NNF and NPF representing the bulk flow percolating through the ventricles. In a magnetic resonance imaging (MRI) study involving 67 multiple sclerosis (MS) patients and 35 healthy controls, Magnano et al. ${ }^{36}$ observed a significant $48 \%$ mean decrease in bulk CSF flow in the patients with MS and a $45 \%$ increase in mean NPF. Mean NNF was also increased in the MS patients, although this was not significant. Similar results were obtained by Gorucu et al., ${ }^{35}$ who also investigated MS patients. However, although these studies associated altered CSF dynamics with MS, they did not observe the venous characteristics of the subjects. By contrast, Zamboni $e t$ $a l .{ }^{8}$ investigated MS patients who were diagnosed with CCSVI. As with the other studies, they observed a large reduction in bulk CSF flow and a tendency towards increased aqueductal pulsatility in MS patients compared with healthy controls. This suggested that in MS patients retrograde venous hypertension in the dural sinuses may be inhibiting absorption of CSF into the SSS, reducing bulk flow and altering aqueductal pulsatility. ${ }^{10}$ This opinion is reinforced by the findings of an interventional study in which venous angioplasty was performed on MS patients with CCSVI. ${ }^{37}$ Prior to the intervention, these patients exhibited increased CSF pulsatility in the AoS, which was lessened when the restricted venous outflow pathways were opened up.

If altered CSF dynamics in patients with MS is due to constricted venous outflow, then one might expect the same phenomenon to be observed in healthy individuals diagnosed with CCSVI. In order to test this hypothesis, Beggs et al. ${ }^{7}$ performed a study on healthy individuals not related to MS patients. The findings of this study were similar to those of Magnano et al. ${ }^{36}$

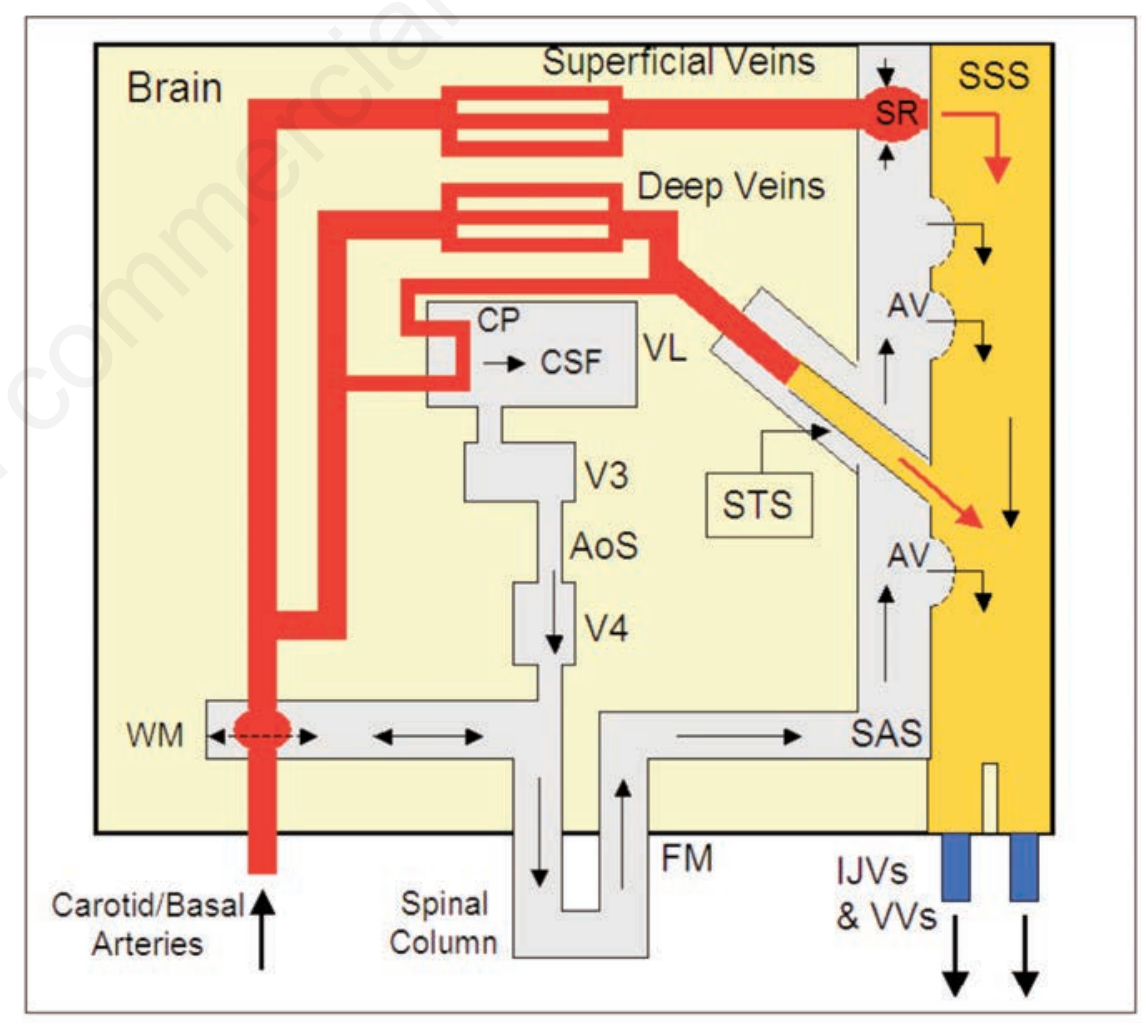

Figure 1. Hydrodynamic model of the intracranial space, showing the interactions between the arterial and venous blood flows and the cerebrospinal fluid (CSF). SSS, superior sagittal sinus; STS, straight sinus; SAS, sub-arachnoid space; AV, arachnoid villi; CP, choroid plexus; FM, foramen magnum; WM, windkessel mechanism; SR, Staling resistor; VL, lateral ventricle; V3, third ventricle; V4, fourth ventricle; AoS, aqueduct of Sylvius; IJVs, internal jugular veins; VVs, vertebral veins. (Courtesy of Biomed Central, the original publisher ${ }^{10}$ ). 
and revealed a statistically significant $32 \%$ increase in CSF NPF in the CCSVI positive subjects, compared with the CCSVI negative individuals, with a tendency towards reduced CSF bulk flow. As such, they suggested that CCSVI is associated with altered CSF dynamics, irrespective of whether on not MS is present, reinforcing the opinion that increased aqueductal CSF pulsatility is primarily a biomechanical phenomenon associated with restricted venous outflow from the cranium.

Increased cerebral blood flow pulsatility has been linked with microstructural white matter (WM) damage. ${ }^{38-40}$ Increased pulsatility in the cerebral vascular bed is indicative of decreased arterial compliance, and is associated with arteriosclerosis $^{41}$ and hypertension. ${ }^{42}$ Hypertension, a known risk factor for small vessel disease $^{43}$ and leukoaraiosis (LA), ${ }^{44}$ is thought to be associated with changes in vascular mechanics. $^{38,42}$ It has been suggested ${ }^{15}$ that increased vascular pulsatility might cause WM damage indicative of early stage LA. ${ }^{39}$ Bateman ${ }^{11}$ found blood flow through the WM to be highly pulsatile in individuals with LA and concluded that this would increase endothelial shear stress, which in turn would cause WM damage. ${ }^{15}$

Jolly et al. ${ }^{39}$ found both increased blood flow pulsatility and increased aqueductal CSF pulse volume to be associated with microstructural WM changes in elderly subjects. Daouk et al. ${ }^{45}$ found apparent diffusion coefficient, an early indicator of microstructural changes, to be strongly correlated with aqueductal stroke vol- ume in Alzheimer's disease (AD) patients. Furthermore, Magnano et al..$^{34}$ found increased aqueductal pulse to be associated with more severe T1 and T2 lesion volumes in MS patients. This raises intriguing questions about the relationship between vascular pulsatility and aqueductal CSF pulsatility. Greitz ${ }^{46}$ postulated a link between increased pulsation in the cerebral vascular bed and CSF pulsatility in the AoS, arguing that pulsations in the cerebral capillaries were transmitted through the parenchyma to the lateral ventricles. However, Beggs et al. ${ }^{7}$ demonstrated that increased aqueductal pulsatility is associated with constricted cerebral venous outflow in healthy adults, suggesting that other mechanisms may be at work. Contrary to Greitz, Beggs argued that impairment of cerebral venous outflow would induce retrograde hypertension in the dural sinuses, reducing intracranial compliance and resulting in altered CSF dynamics. ${ }^{10}$

There is evidence that occlusion of the venous drainage pathways can cause blood to accumulate within the cranium, something that theoretically could alter intracranial compliance. In an experiment involving healthy subjects, Kitano et al. ${ }^{47}$ showed that compression of the internal jugular veins (IJVs) resulted in a 5-20\% increase intracranial blood volume. Frydrychowski et al. ${ }^{18}$ also performed bilateral compression of the IJVs on healthy individuals and found that it caused a reduction in the width of the SAS - a finding consistent with the storage of blood in the cortical veins. Furthermore, in a recent study involving $\mathrm{AD}$

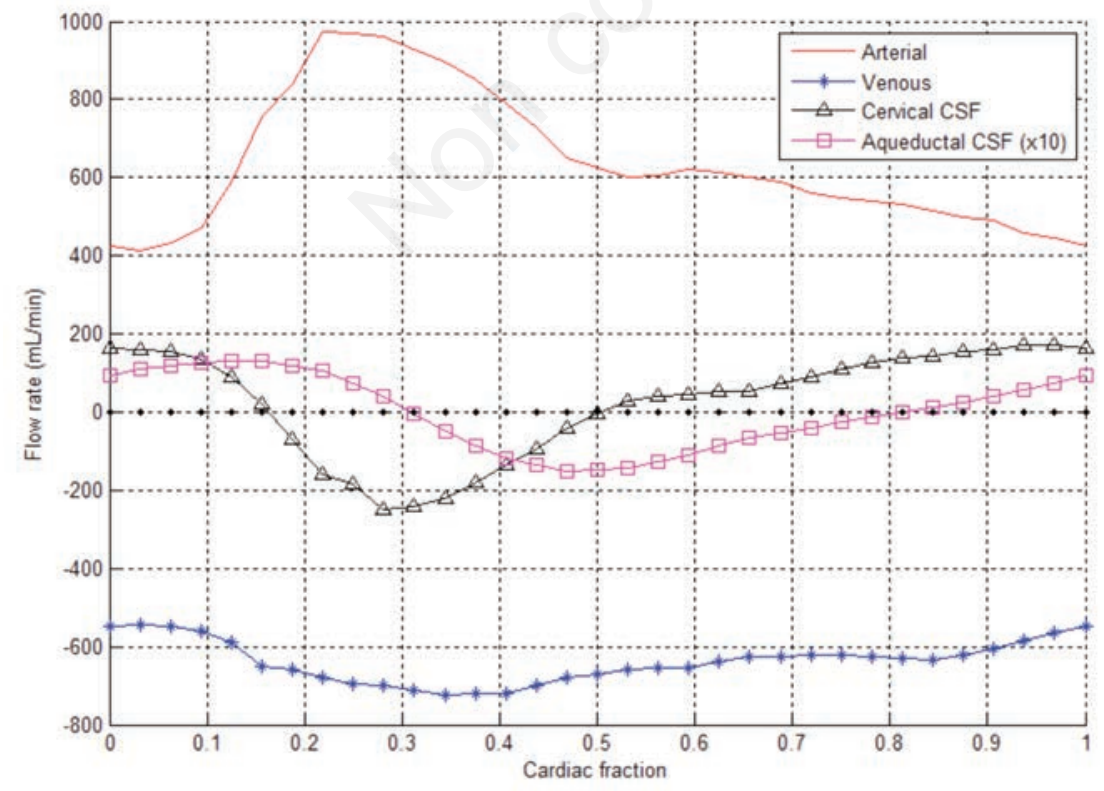

Figure 2. Transient intracranial blood and cerebrospinal fluid (CSF) flow rates over the cardiac cycle in a healthy individual (the figure is based on data published in Ambarki $e t$ al., 200716).

patients, Beggs et al. ${ }^{48}$ found jugular venous reflux to be strongly associated with increased brain parenchyma volume, something that they postulated was possibly due to blood retention within the brain. Because CSF is incompressible, any reduction in the compliance of the cortical bridging veins due to blood retention should, in theory, impact on the windkessel mechanism smoothing blood flow to the cerebral vascular bed. Evidence to support this, comes from the study by Frydrychowski et al. ${ }^{18}$ who observed that during compression of the IJVs, pulsatility in the pial arteries traversing the SAS increased by $107 \%$. Collectively, this suggests that venous drainage anomalies are associated with blood retention in the cerebral veins, and that this in turn is associated with altered biomechanical characteristics within the intracranial space.

\section{Intracranial compliance and venous drainage}

Intracranial compliance is generally characterized by the arteriovenous delay (AVD) between the arterial pulse entering the cranium and the venous pulse leaving it. ${ }^{49}$ One of the major paradoxes of the intracranial fluid system is associated with the AVD. How is this possible, in a system where all the fluids involved are incompressible and the cranium is apparently a rigid container, to have a time lag between the blood flow signals entering and leaving the cranium? The brain parenchyma tissue contains no gaseous material and is generally thought to be incompressible, ${ }^{50}$ due to its very high water content. ${ }^{51}$ One possible explanation to this apparent paradox lies in the cortical bridging veins, which are coupled via the dural sinuses to the extracranial venous drainage system. These collapsible thin walled vessels are thought to play an influential role in regulating intracranial compliance. ${ }^{20,21,52}$ The ability of the cortical veins to store venous blood and delay outflow is dependent on their compliance, with more compliant veins storing greater volumes of blood than incompliant ones. ${ }^{20}$ As a result, compliant veins exhibit greater pulsatility in blood flow. Indeed, Bateman ${ }^{20}$ eloquently showed that in patients with normal pressure hydrocephalus (NPH), cortical vein pulsatility was $60 \%$ less than in the SSS, suggesting that the disease is characterized by a reduction in the compliance of the veins that bridge the SAS. Bateman found that cortical vein compliance was significantly increased following shunt surgery, indicating that the compliance attributed to these vessels is primarily functional, not structural, and dependent on the transmural pressure difference between the venous blood and the sub-arachnoid CSF. This 
implies that the compliance of cortical bridging veins is dependent both on the craniospinal compliance ${ }^{20}$ and the ability of any venous blood stored in them to freely exit the cranium via the extracranial veins. Therefore, any constriction of the extracranial venous drainage pathways could, in theory, influence the compliant behaviour of the cortical veins.

A strong correlation has been demonstrated between intracranial pressure (ICP) and venous pressure in the dural sinuses,$^{53}$ and it has been shown that venous sinus stenting in patients with idiopathic intracranial hypertension (IIH) can rapidly normalize ICP. ${ }^{54}$ While this relationship is poorly understood, there is evidence that the cortical bridging veins play an influential role. ${ }^{20}$ Some have likened the action of the cortical bridging veins to a Starling resistor, which collapses, occluding the blood flow, when the transmural pressure reaches a certain threshold. ${ }^{55}$ The fluid flow through the bridging veins appears not to be regulated by the pressure difference between the two ends of the vessels, but rather by the pressure difference between the blood in the veins and the sub-arachnoid CSF. The cortical bridging veins are very sensitive to small changes in transmural pressure. Because they are required to open and close to regulate blood flow from the cortex, the cortical venous pressure is only about 2 to $5 \mathrm{mmHg}$ higher than the ICP. ${ }^{55}$ This means that small changes in ICP or venous pressure can greatly influence the behavior of blood flow from the cortex. Indeed, it has been estimated that a change of as little as $1.5 \mathrm{mmHg}$ in the difference between ICP and the pressure in the bridging veins could be responsible for the difference between severe hyperemia $(\mathrm{CBF}=1000 \mathrm{~mL} / \mathrm{min})$ to serve ischemia $(\mathrm{CBF}=300 \mathrm{~mL} / \mathrm{min}){ }^{55}$

\section{Postural changes}

Body position is known to have a profound effect on the fluids in the cranium. When upright the pressure in the IJVs becomes subatmospheric, with the result that they collapse. This causes the cerebral venous drainage pathways to be diverted through the vertebral and epidural veins. ${ }^{56}$ Also, when upright the venous pressure at the confluens sinuum in the dural sinuses becomes sub-atmospheric, in adults dropping from a mean of $8.5 \mathrm{mmHg}$ when supine, to $-8.6 \mathrm{mmHg}$ when upright. ${ }^{57}$ The ICP, which is normally in the range 7-15 mmHg when supine, ${ }^{58}$ also falls when upright. Alperin et al..$^{5}$ in an MRI study involving healthy young adults, found that in the upright position there was a reduction in ICP, which fell from a mean of $10.6 \mathrm{mmHg}$ when supine, to $4.5 \mathrm{mmHg}$ when upright. However, others disagree with this finding and instead believe that ICP becomes sub-atmospheric when in the upright position. For example, based on the work of Chapman et al., ${ }^{60}$ Czosnyka and Pickard ${ }^{61}$ concluded that ICP in adults in the vertical position is negative, with a mean of around $-10 \mathrm{mmHg}$.

Given the magnitude of the pressure changes involved in moving from the supine to upright positions, there is reason to believe that this might alter the functional behaviour of the cortical bridging veins and also overall intracranial compliance. Alperin et al..$^{59}$ found that in adults in the upright position, venous outflow became considerably less pulsatile (a $43 \%$ reduction in the venous pulsatility index), with flow occurring predominately through the vertebral plexus, rather than the IJVs, which were the principle drainage pathway when supine. As such, their findings appear to corroborate those of Valdueza et al. ${ }^{56}$ Importantly, Alperin et al also observed a 2.8-fold increase in intracranial compliance when in the upright position compared with supine position, which was associated with 2.4-fold decrease in oscillatory volume of the cervical CSF flow. They also found changing posture to the upright position resulted in a $12 \%$ reduction in CBF. Alperin et al.'s findings are supported by those of Ragauskas et al. ${ }^{62}$ who also observed increased intracranial compliance when in the upright position. While the precise physiological mechanisms involved in the posture-related regulatory process are not understood, these findings appear to be consistent with greatly reduced pressure in the dural sinuses when in the upright position. ${ }^{63}$

\section{Normal pressure hydro- cephalus}

Because increased aqueductal CSF pulsatility appears to be associated with constricted venous outflow, ${ }^{7}$ it is perhaps worth considering $\mathrm{NPH}$ in more detail, a disease that is thought by some $21,49,52,64$ to be associated with venous anomalies and which is characterized by increased aqueductal pulsatility. ${ }^{65-70}$ Normal pressure hydrocephalus occurs when there is an abnormal accumulation of CSF in the ventricles, causing them to become enlarged, ${ }^{71}$ but with little or no increase in ICP. ${ }^{72,73} \mathrm{NPH}$ is associated with significantly reduced CSF absorption through the $\mathrm{AV}$ into the SSS.74,75 Given that ICP does not substantially increase in individuals with $\mathrm{NPH}$, this suggests that CSF is being resorbed elsewhere. ${ }^{76}$ Bateman ${ }^{49}$ postulated that CSF resorption is likely to occur in the subependymal brain parenchyma and some have identified ventricular reflux in NPH patients, ${ }^{77,78}$ leading to oedema and neuronal degeneration. ${ }^{76}$ Tracer studies have shown that CSF can pass through the ependy- mal wall of the ventricles and enter the brain parenchyma. ${ }^{29}$ Tight junctions are absent from most of the ependyma lining the ventricles, making it relatively permeable to the retrograde transport of water, particularly when the CSF pressure is raised. ${ }^{32}$ Trypan blue injected into the CSF in the ventricles readily spreads into the brain, ${ }^{79}$ and tracers injected into the ventricles are taken up by perivascular macrophages, ${ }^{80}$ suggesting that CSF can permeate the perivascular spaces. In hydrocephalus patients, due to impaired drainage of CSF from the ventricles, CSF can pass into the periventricular WM as ventricular reflux causing interstitial edema. ${ }^{81-84}$

Bateman $^{49}$ found the AVD to be $53 \%$ shorter in NPH patients compared with healthy controls. A similar reduction in AVD in NPH patients was observed in a subsequent study, ${ }^{52}$ and Mase et al..$^{85}$ independently confirmed this finding, showing a $64 \%$ reduction in intracranial compliance in NPH patients compared with healthy controls. This suggests that NHP is characterized by reduced intracranial compliance. Bateman ${ }^{20}$ showed that in NPH patients cortical vein pulsatility was $60 \%$ less than in the SSS, indicating a reduction in the compliance of the bridging veins. However, following shunt insertion this situation was reversed and there was a $186 \%$ increase in cortical vein compliance within 3-5 days of the intervention.

Using direct cannulation of the cortical veins, venous sinuses and the SAS in dogs with hydrocephalus, Portnoy et al. ${ }^{86}$ was able to show that the cortical vein-to-CSF pressure difference in hydrocephalic animals was much greater than that in the normal animals. In the hydrocephalic dogs the cortical vein pressure was $21.54 \mathrm{mmHg}$ when the CSF pressure was $16.37 \mathrm{mmHg}$ and the SSS pressure was 8.43 $\mathrm{mmHg}$, compared with respective values of $11.72,10.46$ and $5.15 \mathrm{mmHg}$ in the normal animals. Interestingly, while the hydrocephalic dogs exhibited an increase of only $3.28 \mathrm{mmHg}$ in SSS pressure, this was accompanied by a $9.82 \mathrm{mmHg}$ increase in cortical vein pressure, indicating that hydrocephalus profoundly altered the functional relationship between these two vessels. This suggests that in hydrocephalic patients, the sub-arachnoid CSF may be interacting with cortical bridging veins at their junction with the $\mathrm{SSS},{ }^{20}$ compressing them so that the up-stream venous pressure is greatly increased. Bateman ${ }^{20}$ hypothesized that this increase in cortical venous pressure would be transmitted up-stream to the capillaries resulting in increased production of interstitial fluid. This, together with reduced CSF absorption through the $\mathrm{AV}$, would result in an over production of fluid, which as Bateman demonstrated using nuclear cisternography, might result in retrograde CSF flow in the AoS and ventricular reflux..$^{20}$ 


\section{Hypothesis and perspectives}

From the descusion above it can be seen that while understanding of the intracranial fluid system has improved over the years, much still remains unknown. There is no unifying model which adequately explains the dynamic behaviour of all the component fluids in the intracranial space, and the role of the intracranial fluid system in either preventing, or promoting, neurological disease is poorly understood. In particular, the regulatory role of the cerebral venous system is not well understood. While the contribution of venous anomalies to various neurological pathologies is becoming clearer, ${ }^{10,87}$ much remains to be discovered. For example, there is a need to understand the extent to which venous drainage influences intracranial compliance. If one considers the timing of the peaks in the respective pulses shown in Figure 2, it can be seen that arterial flow into the cranium peaks first, followed closely by the cervical CSF peak in the caudal direction, which is then followed by the peak in venous flow out of the cranium. This indicates that volumetric changes are being rapidly transferred from one fluid to another, which is what one would expect from a system containing non-compressible materials. Having said this, the presence of an AVD indicates that compliance must exist somewhere in the system. While the mechanisms involved are poorly understood, the time delay between the arterial and venous peak flows is likely to be due to a combination of spinal column compliance and the ability of the cortical bridging veins to freely expel stored blood from the cranium via the dural sinuses and extracranial venous pathways. However, while this is a plausible explanation, there is paucity of good quality data on the subject and there is need to better characterize the functional behaviour of the cortical bridging veins both in healthy individuals and patients with neurological conditions. A better understanding of the interaction between the CSF, the bridging veins and the SSS should enable new insights to be gained into the pathophysiology of conditions such as NPH and IIH.

From Figure 2 it can be seen that when the cervical CSF flow reverses during diastole and starts to flow back into the cranium, two things happen: firstly, the volume of arterial blood entering the cranium starts to fall, reducing the volume of blood in the pial arteries; and secondly, the volume of venous blood exiting the cranium also starts to fall, indicating that venous blood is being stored in somewhere in the cranium, presumably in the compliant cortical veins. Given that positive aqueductal flow, towards the lateral ventricles, occurs late in diastole, this suggests that the venous pulse is likely to influence the dynamics of the CSF flow in the AoS. Although, the mechanics of this relationship are not understood, there is good reason to believe that the two pulses might be connected. Nakagawa et al..$^{25}$ and oth$\mathrm{ers}^{22,23}$ all observed the pulsatile compression of cortical bridging veins by the sub-arachnoid CSF, suggesting that the venous signal strongly reflects transient volumetric changes in the cortical bridging veins and thus the overall volume and compliance of the SAS. ${ }^{21,49,64}$ Given that the SAS is a relatively large volume, with low resistance to CSF flow, ${ }^{26}$ it is therefore reasonable to assume that the CSF returning to the cranium during diastole will first tend to fill the SAS, before forcing its way up the relatively high resistance AoS towards the third ventricle. This can be clearly seen in the lag between the cervical and aqueductal CSF signals in Figure 2. The fact that the aqueductal CSF pulse lags the cervical CSF pulse by 0.2 to 0.3 of a cardiac cycle suggests that its dynamic is influence by the compliance of the SAS. Evidence supporting this opinion comes Beggs et al., ${ }^{7}$ who found that constricted venous outflow was strongly associated with increased aqueductal pulsatility healthy adults. The hydraulic resistance of the extracranial venous drainage system has been shown to be on average $63.5 \%$ greater in MS patients diagnosed with CCSVI compared with CCSVI negative healthy controls. ${ }^{2}$ If constriction of the venous drainage pathways inhibits free egress of blood transiently stored in the cortical bridging veins, then this is likely to reduce the compliance of the whole SAS. This would mean that there would be less room to accommodate the returning CSF in the SAS, with the result that more of the fluid would be forced up the AoS towards the third ventricle, which is exactly what Beggs et al. observed. Similar, results have also been observed in MS patients $8,35,36$. Furthermore, Zivadinov et al., ${ }^{37}$ who performed venous angioplasty on MS patients diagnosed with CCSVI, found that the procedure normalized the CSF pulsatility in the AoS, adding weight to the argument that the functional compliance of the cortical bridging veins profoundly influences the dynamics of the aqueductal CSF pulse.

The degree to which constriction of the extracranial venous pathways produces retrograde venous hypertension in the dural sinuses is also not well understood. Given that the pressure drop through the extracranial venous system is normally of the order $3-5 \mathrm{mmHg},{ }^{88}$ an increase of $63 \%$ in the resistance of these vessels (as calculated by Beggs et al. ${ }^{2}$ ) would equate to a pressure increase in the region $1.89-3.15 \mathrm{mmHg}$, assuming that the blood flow rate remains constant. Although only a rough estimation, this calculation is consistent with the $2.21 \mathrm{mmHg}$ mean increase in venous pressure measured in CCSVI positive MS patients by Zamboni et al. ${ }^{89}$ As such, it suggests that
CCSVI is associated with mild venous hypertension $(<5 \mathrm{mmHg})$ in the dural sinuses; something that would tend to reduce absorption of CSF by the $\mathrm{AV}^{26,27}$ and inhibit the bulk flow of $\mathrm{CSF}^{7,8}$

Body position is known to be an important factor affecting ICP. Mavrocordatos et al..$^{90}$ showed that in anaesthetized neurosurgical patients lying on a flat surface, the ICP could be raised (mean increase) by $2.8-3.1 \mathrm{mmHg}$ through simply flexion of the head to left or right, while rotating the head resulted in an mean increase of 4.1-4.8 mmHg. While the reasons for these changes are not fully understood, there is evidence that rotation of the head can compress both the jugular veins and the vertebral veins ${ }^{91}$ inhibiting the cerebral venous drainage. Iwabuchi et al. ${ }^{57}$ investigated changes in venous pressure in the confluens sinuum associated with neck rotation and found that in the supine position, a mean increase of $30.3 \%$ was observed on a rightward rotation, whereas only a mean elevation of $1.1 \%$ was observed for a leftward rotation. However rather surprisingly, in the sitting position, right and left rotations of the neck resulted in increases in pressure of $85.5 \%$ and $18.2 \%$ respectively. Collectively, these findings suggest that the cerebral venous drainage system plays an influential role in regulating ICP. Furthermore, they indicate that the functional behaviour of the cerebral venous drainage system is greatly influenced by postural changes. It is therefore surprising that relatively little is known about how changes in posture (e.g. supine to upright) affect the intracranial fluid system, particularly in healthy individuals, who for ethical reasons are rarely studied. The MRI work by Alperin et al..$^{59}$ revealed marked changes in the behaviour of the intracranial fluid system when healthy subjects move from the supine to upright position. These changes were particularly obvious in the behaviour of the venous system, which became much less pulsatile when upright, something that appears to be associated with greater intracranial compliance in this position.

\section{Clinical relevance}

The issue of cerebral venous drainage has for many years been overlooked and it is only recently that the subject has received much attention. The mystery surrounding its apparent connection with the CSF system, only serves to highlight that relatively little is known about the physiological mechanisms that regulate the intracranial fluid system. In particular, the way in which the intracranial fluid system adapts when changing from supine to the upright position is poorly understood. However, there is evidence that 
impaired cerebral venous outflow can markedly alter the dynamics of the intracranial fluid system. A better understanding of the physiology associated with cerebral venous outflow may therefore be of great benefit in understanding the progression of neurological conditions such as NPH and IIH.

\section{Conclusions}

There is growing evidence that the cerebral venous drainage plays an influential role in regulating the dynamics of the intracranial fluid system. In particular, the compliance of the cortical bridging veins appears to be critical to the behaviour of the system, with abnormalities at this location implicated in NPH. The compliance associated with these vessels appears to be functional in nature and dependent on the free egress of blood out of the cranium via the extracranial venous drainage pathways. Constricted venous outflow appears to be linked to increased CSF pulsatility in the AoS, suggesting that inhibited venous blood flow may be altering the compliance of the cortical bridging veins.

\section{References}

1. Zamboni P, Galeotti R, Menegatti E, et al. Chronic cerebrospinal venous insufficiency in patients with multiple sclerosis. $\mathrm{J}$ Neurol Neurosurg Psychiatry 2009;80:3929.

2. Beggs C, Shepherd S, Zamboni P. Cerebral venous outflow resistance and interpretation of cervical plethysmography data with respect to the diagnosis of chronic cerebrospinal venous insufficiency. Phlebology 2014;29:191-9.

3. Zamboni P, Menegatti E, Occhionorelli S, Salvi F. The controversy on chronic cerebrospinal venous insufficiency. Veins and Lymphatics 2013;2:e14.

4. Doepp F, Paul F, Valdueza JM, et al. No cerebrocervical venous congestion in patients with multiple sclerosis. Ann Neurol 2010;68:173-83.

5. Beggs C. Multiple sclerosis appears to be associated with cerebral venous abnormalities. Ann Neurol 2010;68:560-1; author reply $561-2$.

6. Khan 0, Filippi M, Freedman MS, et al. Chronic cerebrospinal venous insufficiency and multiple sclerosis. Ann Neurol 2010;67:286-90.

7. Beggs CB, Magnano C, Shepherd SJ, et al. Aqueductal cerebrospinal fluid pulsatility in healthy individuals is affected by impaired cerebral venous outflow. J Magn
Reson Imaging 2014;40:1215-22.

8. Zamboni P, Menegatti E, WeinstockGuttman B, et al. The severity of chronic cerebrospinal venous insufficiency in patients with multiple sclerosis is related to altered cerebrospinal fluid dynamics. Funct Neurol 2009;24:133-8.

9. Zivadinov R, Weinstock-Guttman B. Funding CCSVI research is/was a waste of valuable time, money and intellectual energy: no. Mult Scler 2013;19:858-60.

10. Beggs CB. Venous hemodynamics in neurological disorders: an analytical review with hydrodynamic analysis. BMC Med 2013;11:142.

11. Bateman GA. Pulse-wave encephalopathy: a comparative study of the hydrodynamics of leukoaraiosis and normal-pressure hydrocephalus. Neuroradiology 2002;44:740-8.

12. Egnor M, Rosiello A, Zheng L. A model of intracranial pulsations. Pediatr Neurosurg 2001;35:284-98.

13. Wagshul ME, Eide PK, Madsen JR. The pulsating brain: A review of experimental and clinical studies of intracranial pulsatility. Fluids Barriers CNS 2011;8:5.

14. Egnor M, Zheng L, Rosiello A, et al. A model of pulsations in communicating hydrocephalus. Pediatr Neurosurg 2002;36:281303.

15. Bateman GA, Levi CR, Schofield P, et al. The venous manifestations of pulse wave encephalopathy: windkessel dysfunction in normal aging and senile dementia. Neuroradiology 2008;50:491-7.

16. Ambarki K, Baledent 0, Kongolo G, et al. A new lumped-parameter model of cerebrospinal hydrodynamics during the cardiac cycle in healthy volunteers. IEEE Trans Biomed Eng 2007;54:483-91.

17. Ivanov AY, Petrov AE, Vershinina EA, et al. Evidence of active regulation of cerebral venous tone in individuals undergoing embolization of brain arteriovenous malformations. J Appl Physiol (1985) 2013;115:1666-71.

18. Frydrychowski AF, Winklewski PJ, Guminski W. Influence of acute jugular vein compression on the cerebral blood flow velocity, pial artery pulsation and width of subarachnoid space in humans. PLoS One 2012;7:e48245.

19. An H, Lin W. Cerebral venous and arterial blood volumes can be estimated separately in humans using magnetic resonance imaging. Magn Reson Med 2002;48:583-8.

20. Bateman GA. The reversibility of reduced cortical vein compliance in normal-pressure hydrocephalus following shunt insertion. Neuroradiology 2003;45:65-70.

21. Williams H. A unifying hypothesis for hydrocephalus, Chiari malformation, syringomyelia, anencephaly and spina bifida. Cerebrospinal Fluid Res 2008;5:7.
22. Luce JM, Huseby JS, Kirk W, Butler J. A Starling resistor regulates cerebral venous outflow in dogs. J Appl Physiol 1982;53:1496-503.

23. Vignes JR, Dagain A, Guerin J, Liguoro D. A hypothesis of cerebral venous system regulation based on a study of the junction between the cortical bridging veins and the superior sagittal sinus. Laboratory investigation. J Neurosurg 2007;107:1205-10.

24. Greitz D. Cerebrospinal fluid circulation and associated intracranial dynamics. A radiologic investigation using MR imaging and radionuclide cisternography. Acta Radiol Suppl 1993;386:1-23.

25. Nakagawa Y, Tsuru M, Yada K. Site and mechanism for compression of the venous system during experimental intracranial hypertension. J Neurosurg 1974;41:427-34.

26. Ekstedt J. CSF hydrodynamic studies in man. 2. Normal hydrodynamic variables related to CSF pressure and flow. J Neurol Neurosurg Psychiatry 1978;41:345-53.

27. Cutler RW, Page L, Galicich J, Watters GV. Formation and absorption of cerebrospinal fluid in man. Brain 1968;91:707-20.

28. Ekstedt J. CSF hydrodynamic studies in man. 1. Method of constant pressure CSF infusion. J Neurol Neurosurg Psychiatry 1977;40:105-19.

29. Weller RO, Djuanda E, Yow HY, Carare RO. Lymphatic drainage of the brain and the pathophysiology of neurological disease. Acta Neuropathol 2009;117:1-14.

30. Cserr HF, Knopf PM. Cervical lymphatics, the blood-brain barrier and the immunoreactivity of the brain: a new view. Immunol Today 1992;13:507-12.

31. Johanson CE, Duncan JA 3rd, Klinge PM, et al. Multiplicity of cerebrospinal fluid functions: new challenges in health and disease. Cerebrospinal Fluid Res 2008;5 :10.

32. Abbott NJ. Evidence for bulk flow of brain interstitial fluid: significance for physiology and pathology. Neurochem Int 2004; $45: 545-52$.

33. Lorenzo AV, Page LK, Watters GV. Relationship between cerebrospinal fluid formation, absorption and pressure in human hydrocephalus. Brain 1970;93:67992.

34. Magnano C, Schirda C, Weinstock-Guttman B, et al. Cine cerebrospinal fluid imaging in multiple sclerosis. J Magn Reson Imaging 2012;36:825-34.

35. Gorucu Y, Albayram S, Balci B, et al. Cerebrospinal fluid flow dynamics in patients with multiple sclerosis: a phase contrast magnetic resonance study. Funct Neurol 2011;26:215-22.

36. Magnano C, Schirda C, Weinstock-Guttman B, et al. Cine cerebrospinal fluid imaging in multiple sclerosis. J Magn Reson Imaging 2012;36:825-34. 
37. Zivadinov R, Magnano C, Galeotti R, et al. Changes of cine cerebrospinal fluid dynamics in patients with multiple sclerosis treated with percutaneous transluminal angioplasty: case-control study. J Vasc Interv Radiol 2013;24:829-38.

38. Mitchell GF, van Buchem MA, Sigurdsson S, et al. Arterial stiffness, pressure and flow pulsatility and brain structure and function: the age, gene/environment susceptibility - Reykjavik study. Brain 2011;134: 3398-407.

39. Jolly TA, Bateman GA, Levi CR, et al. Early detection of microstructural white matter changes associated with arterial pulsatility. Front Hum Neurosci 2013;7:782.

40. Wahlin A, Ambarki K, Birgander R, et al. Intracranial pulsatility is associated with regional brain volume in elderly individuals. Neurobiol Aging 2014;35:365-72.

41. Henry-Feugeas MC. Intracranial MR dynamics in clinically diagnosed Alzheimer's disease: the emerging concept of "pulse wave encephalopathy". Curr Alzheimer Res 2009;6:488-502.

42. Safar ME, Levy BI, Struijker-Boudier H. Current perspectives on arterial stiffness and pulse pressure in hypertension and cardiovascular diseases. Circulation 2003;107:2864-9.

43. Gons RA, de Laat KF, van Norden AG, et al. Hypertension and cerebral diffusion tensor imaging in small vessel disease. Stroke 2010;41:2801-6.

44. van Gijn J. Leukoaraiosis and vascular dementia. Neurology 1998;51:S3-8.

45. Daouk J, Chaarani B, Zmudka J, et al. Relationship between cerebrospinal fluid flow, ventricles morphology, and DTI properties in internal capsules: differences between Alzheimer's disease and normalpressure hydrocephalus. Acta Radiol 2013. [Epub ahead of print].

46. Greitz D. Radiological assessment of hydrocephalus: new theories and implications for therapy. Neurosurg Rev 2004;27:14565; discussion 166-7.

47. Kitano M, Oldendorf WH, Cassen B. The elasticity of the cranial blood pool. J Nucl Med 1964;5:613-25.

48. Beggs C, Chung CP, Bergsland N, et al. Jugular venous reflux and brain parenchyma volumes in elderly patients with mild cognitive impairment and Alzheimer's disease. BMC Neurol 2013;13:157.

49. Bateman GA. Vascular compliance in normal pressure hydrocephalus. AJNR Am J Neuroradiol 2000;21:1574-85.

50. Miller K, Chinzei K. Constitutive modelling of brain tissue: experiment and theory. $\mathrm{J}$ Biomechanics 1997;30:1115-21.

51. Bilston LE. Brain tissue mechanical properties. In: Miller K, ed. Biomechanics of the brain. New York: Springer; 2011. pp 69-89.
52. Bateman GA. The pathophysiology of idiopathic normal pressure hydrocephalus: cerebral ischemia or altered venous hemodynamics? AJNR Am J Neuroradiol 2008;29:198-203.

53. Pickard JD, Czosnyka Z, Czosnyka M, et al. Coupling of sagittal sinus pressure and cerebrospinal fluid pressure in idiopathic intracranial hypertension-a preliminary report. Acta Neurochir Suppl 2008;102: 283-5.

54. Fargen KM, Velat GJ, Lewis SB, et al. Concomitant intracranial pressure monitoring during venous sinus stenting for intracranial hypertension secondary to venous sinus stenosis. J Neurointerv Surg 2013;5:e22.

55. Schaller B. Physiology of cerebral venous blood flow: from experimental data in animals to normal function in humans. Brain Res Brain Res Rev 2004;46:243-60.

56. Valdueza JM, von Munster T, Hoffman 0, et al.Postural dependency of the cerebral venous outflow. Lancet 2000;355:200-1.

57. Iwabuchi T, Sobata E, Ebina K, et al. Dural sinus pressure: various aspects in human brain surgery in children and adults. Am J Physiol 1986;250:H389-96.

58. Albeck MJ, Borgesen SE, Gjerris F, et al. Intracranial pressure and cerebrospinal fluid outflow conductance in healthy subjects. J Neurosurg 1991;74:597-600.

59. Alperin N, Lee SH, Sivaramakrishnan A, Hushek SG. Quantifying the effect of posture on intracranial physiology in humans by MRI flow studies. J Magn Reson Imaging 2005;22:591-6.

60. Chapman PH, Cosman ER, Arnold MA. The relationship between ventricular fluid pressure and body position in normal subjects and subjects with shunts: a telemetric study. Neurosurgery 1990;26:181-9.

61. Czosnyka M, Pickard JD. Monitoring and interpretation of intracranial pressure. J Neurol Neurosurg Psychiatry 2004;75:81321.

62. Ragauskas A, Daubaris G, Petkus V, et al. Clinical study of craniospinal compliance non-invasive monitoring method. Acta Neurochir Suppl 2008;102:165-9.

63. Hicks JW, Munis JR. The siphon controversy counterpoint: the brain need not be "baffling". Am J Physiol Regul Integr Comp Physiol 2005;289:R629-32.

64. Williams $H$. The venous hypothesis of hydrocephalus. Med Hypotheses 2008;70: 743-7.

65. Luetmer PH, Huston J, Friedman JA, et al. Measurement of cerebrospinal fluid flow at the cerebral aqueduct by use of phasecontrast magnetic resonance imaging: technique validation and utility in diagnosing idiopathic normal pressure hydrocephalus. Neurosurgery 2002;50:534-43; discussion 543-4.

66. Schroth G, Klose U. Cerebrospinal fluid flow. III. Pathological cerebrospinal fluid pulsations. Neuroradiology 1992;35:16-24.

67. Gideon P, Stahlberg F, Thomsen C, et al. Cerebrospinal fluid flow and production in patients with normal pressure hydrocephalus studied by MRI. Neuroradiology 1994;36:210-5.

68. Kim DS, Choi JU, Huh R, et al. Quantitative assessment of cerebrospinal fluid hydrodynamics using a phase-contrast cine MR image in hydrocephalus. Childs Nerv Syst 1999;15:461-7.

69. El Sankari S, Gondry-Jouet C, Fichten A, et al. Cerebrospinal fluid and blood flow in mild cognitive impairment and Alzheimer's disease: a differential diagnosis from idiopathic normal pressure hydrocephalus. Fluids Barriers CNS 2011;8:12.

70. Bradley WG Jr, Scalzo D, Queralt J, et al. Normal-pressure hydrocephalus: evaluation with cerebrospinal fluid flow measurements at MR imaging. Radiology 1996;198:523-9.

71. Kitagaki H, Mori E, Ishii K, et al. CSF spaces in idiopathic normal pressure hydrocephalus: morphology and volumetry. AJNR Am J Neuroradiol 1998;19:1277-84.

72. Kiefer M, Unterberg A. The differential diagnosis and treatment of normal-pressure hydrocephalus. Dtsch Arztebl Int;109:15-25; quiz 26.

73. Tsunoda A, Mitsuoka $\mathrm{H}$, Bandai $\mathrm{H}$, et al. Intracranial cerebrospinal fluid measurement studies in suspected idiopathic normal pressure hydrocephalus, secondary normal pressure hydrocephalus, and brain atrophy. J Neurol Neurosurg Psychiatry 2002;73:552-5.

74. Tullberg M, Mansson JE, Fredman P, et al. CSF sulfatide distinguishes between normal pressure hydrocephalus and subcortical arteriosclerotic encephalopathy. J Neurol Neurosurg Psychiatry 2000;69:7481.

75. Bradley WG. Normal pressure hydrocephalus: new concepts on etiology and diagnosis. AJNR Am J Neuroradiol 2000; 21:1586-90

76. Tullberg M, Jensen C, Ekholm S, Wikkelso C. Normal pressure hydrocephalus: vascular white matter changes on MR images must not exclude patients from shunt surgery. AJNR Am J Neuroradiol 2001;22: 1665-73.

77. Brumback RA, Yoder FW, Andrews AD, et al. Normal pressure hydrocephalus. Recognition and relationship to neurological abnormalities in Cockayne's syndrome. Arch Neurol 1978;35:337-45.

78. Algin 0, Hakyemez B, Ocakoglu G, Parlak M. MR cisternography: is it useful in the diagnosis of normal-pressure hydro- 
cephalus and the selection of "good shunt responders"? Diagn Interv Radiol 2011;17:105-11.

79. Goldman EE. Vitalfarbung am Zentralnervensystem. Abh Preuss Akad Wiss Phys Math K1 1913;1:1-60.

80. Bechmann I, Kwidzinski E, Kovac AD, et al. Turnover of rat brain perivascular cells. Exp Neurol 2001;168:242-9.

81. Weller R0, Massey A, Newman TA, et al. Cerebral amyloid angiopathy: amyloid beta accumulates in putative interstitial fluid drainage pathways in Alzheimer's disease. Am J Pathol 1998;153:725-33.

82. Weller R0, Wisniewski H. Histological and ultrastructural changes with experimental hydrocephalus in adult rabbits. Brain 1969;92:819-28.

83. Wisniewski H, Weller R0, Terry RD. Experimental hydrocephalus produced by the subarachnoid infusion of silicone oil. J Neurosurg 1969;31:10-4.

84. Weller RO, Wisniewski H, Shulman K, Terry RD. Experimental hydrocephalus in young dogs: histological and ultrastructural study of the brain tissue damage. J Neuropathol Exp Neurol 1971;30:613-26.

85. Mase M, Miyati T, Kasai $H$, et al. Noninvasive estimation of intracranial compliance in idiopathic NPH using MRI. Acta Neurochir Suppl 2008;102:115-8.

86. Portnoy HD, Branch C, Castro ME. The relationship of intracranial venous pressure to hydrocephalus. Childs Nerv Syst 1994;10: 29-35.

87. Haacke EM, Beggs CB, Habib C. The role of venous abnormalities in neurological disease. Rev Recent Clin Trials 2012;7:100-16.

88. Dawson EA, Secher NH, Dalsgaard MK, et al. Standing up to the challenge of stand- ing: a siphon does not support cerebral blood flow in humans. Am J Physiol Regul Integr Comp Physiol 2004;287:R911-4.

89. Zamboni P, Galeotti R, Menegatti E, et al. A prospective open-label study of endovascular treatment of chronic cerebrospinal venous insufficiency. J Vasc Surg 2009;50: 1348-58e1-3.

90. Mavrocordatos P, Bissonnette B, Ravussin P. Effects of neck position and head elevation on intracranial pressure in anaesthetized neurosurgical patients: preliminary results. J Neurosurg Anesthesiol 2000;12:10-4.

91. Hulme A, Cooper R. The effect of head position and jugular vein compression on ICP. A clinical study. Intracranail Pressure III. Berlin: Springer-Verlag; 1976. pp 259263. 